\title{
Die Wirklichkeit der Public Relations
}

\author{
Olaf Hoffjann
}

Public Relations und Wirklichkeit werden seit jeher spannungsreiche Beziehungen unterstellt. Die einen erwarten normativ, dass $P R$ wabrhaftig sein solle. Andere nebmen an, dass PR Themen und Ereignisse mit Blick auf die Wirkung auswäble und idealisierend darstelle. Dritte unterstellen wiederum der PR, dass sie (immer) täusche und (mitunter) lüge. Die Annabmen der verschiedenen erkenntnistheoretischen Perspektiven sind so unterschiedlich wie die Fragen, für die sie sich interessieren. In dem Beitrag werden die relevanten Unterschiede von drei erkenntnistheoretischen Ansätzen zur Wirklichkeit der $P R$ herausgearbeitet. Dabei stehen die folgenden Fragen im Mittelpunkt: Warum bzw. woran scheitern PR-Beschreibungen? Was ist das zentrale Erfolgskriterium für PR-Beschreibungen? Und: Wie werden Entwicklungen wie die Fiktionalisierung oder die Zunabme von Inszenierungen erklärt? Die erkenntnistheoretischen Kandidaten sind das realistische und konstruktivistische Paradigma sowie die non-dualistische Perspektive. Es wird gezeigt, dass sich das realistische und das konstruktivistische Paradigma einige Fragen gar nicht stellen, andere nicht plausibel beantworten können. Eine vielversprechende Alternative scheint der Non-Dualismus zu sein, der durch seinen Verzicht auf eine ontische Realität erkenntnistheoretische Fallen vermeidet.

Schlagwörter: Public Relations, Erkenntnistheorie, Realismus, Konstruktivismus, Non-Dualismus

Wenn im schwierigen Verhältnis der PR zur Wahrheit der Schuldige gesucht wird, sind die Rollen meist klar verteilt. So wird die internationale PR-Agentur Hill \& Knowlton für die heute als fiktiv bezeichnete Geschichte der Baby-Morde 1991 in Kuwait verantwortlich gemacht. Dem tränenreichen wie später als gelogen bezeichneten Bericht der vermeintlichen Krankenschwester wird zugeschrieben, dass er der letzte Auslöser für den Eintritt der USA in den Krieg gegen den Irak war (vgl. Kunczik 2002: 36). Kurzfristig mag die Agentur mit ihrer Version erfolgreich gewesen sein - und vielleicht hatte sie auch nur den kurzfristigen Erfolg im Blick -, langfristig ist die „Wahrheit“ dann doch publik geworden. Mitunter sind die Rollen weniger klar verteilt: Als Shell 1995 die Ölplattform Brent Spar versenken wollte, behauptete der Konzern, dass sich noch etwa 150 Tonnen Schadstoffe in der Ölplattform befänden. Greenpeace bezeichnete diese Angabe als falsch, nannte die Zahl von rund 5.500 Tonnen und setzte sich damit in der Medienberichterstattung weitgehend durch. Nach dem Ende der öffentlichen Auseinandersetzung nannte eine Expertenkommission schließlich eine Menge zwischen 74,3 und 102,7 Tonnen, die bis heute kaum mehr hinterfragt wird (vgl. Shell 1995; Vorfelder 1995).

Warum wird mitunter eine vermeintliche Lüge geglaubt, während in anderen Fällen die vermeintliche Wahrheit nicht geglaubt wird? Während Realisten mit Blick auf den Ausgang der beiden Fälle vermutlich zufrieden konstatieren würden, dass sich am Ende doch „die“ Wahrheit durchgesetzt habe, könnten Konstruktivisten lapidar darauf hinweisen, dass Fiktionen in einer Mediengesellschaft zunehmend wichtiger werden und ihre Nichtnachprüfbarkeit akzeptiert werde (vgl. Merten 1992: 37). Letztlich bleiben beide Perspektiven unbefriedigend. Denn einerseits erscheinen angesichts zahlreicher Inszenierungen auf öffentlichen, halb-öffentlichen und nicht-öffentlichen Vorder- und Hinterbühnen realistische Annahmen unterkomplex, dass man „die“ Realität erkennen 
könnte. Andererseits gerät die konstruktivistische Perspektive an ihre Grenzen, wenn sie zwischen der epistemologischen Allaussage - alles ist konstruiert - und der Trendhypothese - es wird immer mehr konstruiert - zu differenzieren versucht (vgl. Weber 2005: 333).

Deshalb soll hier eine dritte erkenntnistheoretische Perspektive geprüft werden. Dieser dritte Weg ist der Non-Dualismus, den der österreichische Philosoph Mitterer ausgearbeitet $(1992,2001)$ und zu dem Weber eine Medientheorie entwickelt hat (2005). Mit seiner non-dualistischen Perspektive wendet sich Mitterer insbesondere vom Konstruktivismus und vom Realismus ab, indem er auf beschreibungsverschiedene Objekte bzw. den Bezug auf eine ontische Realität völlig verzichtet. Beschreibungen - so genannte Beschreibungen from now on - führen immer bereits geleistete Beschreibungen fort - so genannte Beschreibungen so far. Erkenntnis ist im Non-Dualismus „nach wie vor Erkenntnis von etwas, von Wirklichkeit. Nur wird das Objekt der Erkenntnis oder der Beschreibung nicht als factum brutum im Jenseits der Diskurse angesiedelt, sondern als bereits in irgendeiner Weise Erkanntes und Beschriebenes bestimmt" (Schmidt 2010: 95). Im Gegensatz zum Konstruktivismus und Realismus, die Mitterer als dualistische Ansätze bezeichnet, nähern sich in einer non-dualistischen Perspektive Beschreibungen damit nie dem beschreibungsverschiedenen Objekt an. Es gibt keine beschreibungsverschiedenen Objekte mehr. Damit verzichtet Mitterer auf eine beschreibungsverschiedene Wirklichkeit - er lengnet sie nicht. Rund 20 Jahre nach Erscheinen von Mitterers Dissertation beschränkt sich die Rezeption des Non-Dualismus in den Kommunikationsund Medienwissenschaften immer noch auf wenige Autoren (vgl. zur Diskussion Riegler/Weber 2008, 2010), die in zwei Richtungen unterteilt werden können. Während sich Weber in seinen Arbeiten eng an Mitterer orientiert und auf dessen Grundlage eine nondualistische Medientheorie (2005) ausarbeitet, werden Mitterers Arbeiten von anderen Autoren genutzt, um konstruktivistische Positionen - z. T. unter neuem Namen - weiterzuentwickeln bzw. zu schärfen (z. B. Schmidt 2003).

Diese einführenden Erläuterungen zum Non-Dualismus sollen an dieser Stelle genügen, da in dem Beitrag nicht der Non-Dualismus zur Diskussion steht, sondern die Frage, welche relevanten $P R$-Phänomene eine non-dualistische Perspektive plausibler erklären kann als eine realistische bzw. eine konstruktivistische. Dazu sollen den erkenntnistheoretischen Kandidaten drei Fragen gestellt werden:

1. Warum bzw. woran scheitern PR-Beschreibungen? Dies ist letztlich die erkenntnistheoretische Kernfrage nach den Bedingungen bzw. Möglichkeiten des Erkennens. Mit dieser Frage hängt eng die zweite zusammen:

2. Was ist das zentrale Erfolgskriterium für PR-Beschreibungen? PR hat als Selbstdarstellungskommunikation im Vergleich zu journalistischen Fremdbeschreibungen einen erheblichen Glaub- bzw. Vertrauenswürdigkeitsnachteil (vgl. Bentele/Seidenglanz 2004: 79). Daraus folgt die Frage, wie PR es gelingt, dass ihre Beschreibungen (mitunter) dennoch geglaubt werden.

3. Wie werden Trend-Beobachtungen wie die Fiktionalisierung oder die Zunabme von Inszenierungen erklärt? PR als strategische Kommunikation inszeniert Ereignisse und Botschaften und veröffentlicht mithin Fiktionen (vgl. Westerbarkey 2004). Daher braucht eine erkenntnistheoretische Perspektive zur Wirklichkeit der PR ein plausibles Verständnis dieser Trend-Beobachtungen.

Mit diesen Fragen befindet sich der Beitrag mitten im erkenntnistheoretischen Diskurs der deutschsprachigen Kommunikationswissenschaft. Der Diskurs nahm in den 90er Jahren eine zentrale Stellung im Fach ein, ist spätestens seit der Münsteraner DGPuKTagung 2001 zum Thema „Fakten und Fiktionen - Über den Umgang mit Medienwirk- 
lichkeiten" (Baum/Schmidt 2002) aber zunehmend wieder zu einem Randthema geworden. Die Auseinandersetzung zwischen Vertretern realistischer und konstruktivistischer Positionen dürfte eine der intensivsten des Faches gewesen sein, „ihre polarisierende und teilweise polemische Form hatte durchaus den Charakter eines reinigenden Gewitters und setzte viel kreatives wissenschaftliches Potenzial frei“ (Scholl 2011: 162). Diese beiden Positionen prägen auch die erkenntnistheoretische Diskussion zur Wirklichkeit der PR. In den vergangenen Jahren ist die Diskussion zwischen realistischen und konstruktivistischen Positionen intensiviert und mitunter erneut polemisch geführt worden (vgl. dazu u. a. Bentele 2009, 2010; Merten 2006, 2010, 2011). Da der Erkenntnisgewinn der jüngeren Beiträge dieser Diskussion selten über frühere Arbeiten hinausgeht, soll aber nur vereinzelt auf jüngere Beiträge eingegangen werden. Es überrascht, dass erkenntnistheoretische Fragen in der PR-Forschung nicht noch intensiver diskutiert wurden, da der Lügenverdacht ein ständiger Begleiter der PR ist. In vielen PR-theoretischen Ansätzen sind sie allenfalls implizit thematisiert worden - so z. B. in systemtheoretischen PR-Theorien durch die spezifische Selektivität der PR.

Abschließend noch ein methodischer Hinweis: Wenn in dem Beitrag PR vor allem aus erkenntnistheoretischer Perspektive erläutert wird, soll hier auf weiterführende sozialtheoretische Einordnungen so weit wie möglich verzichtet werden. So soll nicht erläutert werden, was spezifische Erwartungen an PR sind und worin sie sich z. B. von Erwartungen an den Journalismus unterscheiden. Der Nutzen einer solchen Perspektive liegt darin, dass sie damit offen und anschlussfähig bleibt für verschiedene sozialtheoretische Ansätze von PR. Der Nachteil ist hingegen ohne Zweifel, dass die konkreten Erwartungen an PR und an den „Wahrheitsgehalt“ ihrer Beschreibungen von der jeweiligen gewählten PR-Theorie abhängen. Dies ist in Kauf zu nehmen, wenn im Folgenden der Unterschied zwischen PR und Journalismus im Wesentlichen darauf beschränkt wird, dass PR mitgeteilte Selbstbeobachtungen sind, während Journalismus mitgeteilte Fremdbeobachtungen sind.

\section{Die Ohnmachtsthese im realistischen Paradigma}

Auch wenn heute kein ernst zu nehmender Kommunikationswissenschaftler seinen Ansatz als Realismus bezeichnen würde, prägen realistische Vorstellungen bis heute eine Vielzahl von Beiträgen im Diskurs. Die Folgen dieses realistischen Paradigmas für erkenntnistheoretische Kernfragen der PR sollen am Beispiel der Arbeiten von Bentele diskutiert werden. Bentele hat seinen Ansatz - zunächst zur Medienrealität allgemein (1988, hier: 2008), später auch zur PR-Realität (z. B. 1994b) - entwickelt, um die Frage nach der Glaubwürdigkeit von Medien bzw. der PR zu untersuchen. Zwar grenzt auch Bentele seinen Ansatz, den er als rekonstruktiven Ansatz bezeichnet, selbst von der realistischen Position ab (vgl. Bentele 2008: 257-260). Letztlich kann seine Perspektive aber als realistische Variante interpretiert werden, da sein rekonstruktiver Ansatz im Wesentlichen auf der Annahme basiert, dass die Wirklichkeit bzw. die Realität unendlich viele verschiedene Informationen enthält. Da diese sich nicht vollständig abbilden lassen, wird jeweils ein Teil aktualisiert (vgl. Bentele 1994b: 251), allerdings „nicht beliebig, sondern nach Maßgabe der Muster schon vorhandener objektiver und subjektiver Informationen" (ebd.: 251). Entsprechend sieht Bentele in der Beziehung zwischen Text und außertextlicher Information bzw. Wirklichkeit einen zentralen Unterschied seines Ansatzes zur konstruktivistischen Perspektive (vgl. ebd.: 253).

Bentele hat die Grenzen von PR-Beschreibungen und ihre Erfolgskriterien in zahlreichen Publikationen detailliert beschrieben. Beginnen wir mit der Frage, woran PRBeschreibungen scheitern. PR-Beschreibungen scheitern in Benteles Ansatz letztlich an 
der Realität. Dazu entwickelt Bentele die Diskrepanzthese (vgl. 1994a: 148). Bezugsgruppen können Diskrepanzen zwischen direkt wahrgenommenen Wirklichkeitsausschnitten und Medienwirklichkeiten (Realitätsvergleich) oder zwischen den verschiedenen Medienwirklichkeiten (Medienvergleich) wahrnehmen - eine fehlende Adäquatheit der innerhalb der Medienwirklichkeit enthaltenen Informationen ergeben Indikatoren für die wahrgenommene Glaubwürdigkeit von PR (vgl. Bentele 2005: 157). Entsprechend ist eine Voraussetzung für Glaubwürdigkeit und Vertrauen, dass „eine Art von Repräsentations- oder Isomorphiebeziehung sowie ein Konsistenzverhältnis zwischen PR-Information und zugrunde liegenden Sachverhalten/Ereignissen nicht nur behauptet, sondern tatsächlich gewährleistet ist" (ders. 1992a: 164; vgl. 1994b: 255). Sobald ein bestimmter „Realitäts-,Korridor”“ (ders. 2005: 158) verlassen wird, entstehen Vertrauensprobleme. Die Probleme einer solchen Perspektive sind vielfach am Beispiel der News Bias-Forschung diskutiert worden (vgl. z. B. Schulz 1990: 22-23). Um Verzerrungen durch die Medien zu belegen, wurden Extra-Media-Daten mit Intra-Media-Daten verglichen (z. B. Rosengren 1979). Erkenntnistheoretisch problematisch ist dies, weil auch die Extra-Media-Daten Konstruktionscharakter besitzen. Damit werden in realistischen Ansätzen die Konstruiertheit, die konsequente beobachterbezogene Perspektivierung sowie die selbstreferenzielle und differenzielle Logik nicht berücksichtigt (vgl. Scholl 2002: 10).

Daraus folgen unmittelbar die zentralen Erfolgskriterien für PR-Beschreibungen. Weil Bentele Realitätsvergleiche für möglich hält (vgl. 1994b: 254), ist eine realistisch verstandene Wahrheit das zentrale Erfolgskriterium für PR-Beschreibungen. Damit unterscheiden sich PR-Beschreibungen letztlich kaum oder gar nicht von journalistischen Beschreibungen; für beide gelten die gleichen Adäquatheitsregeln (vgl. ders. 1992b: 42). Daraus folgt, dass Bentele sehr optimistisch ist, dass die Wahrheitsnorm in der Regel auch eingehalten wird. Denn weil Lügen, Übertreibungen o. Ä. durch Realitätsvergleiche leicht zu entlarven sind, wären z. B. Vertrauensverluste kaum zu vermeiden. Ein Festhalten an der Wahrheitsnorm ist damit für PR-treibende Organisationen „überlebenswichtig für das Funktionieren und die Bestandserhaltung“ (ders. 2009: 25). Benteles Position, die von vielen Autoren zumindest implizit geteilt wird, kann daher als Ohnmachtsthese der PR bezeichnet werden, weil sie den Spielraum von PR durch die Möglichkeit eines Realitätsvergleiches eng begrenzt.

Wie werden im realistischen Paradigma schließlich Trend-Beobachtungen wie die Fiktionalisierung oder die Zunabme von Inszenierungen erklärt? Letztlich spielen diese Trends in einer realistischen Perspektive keine Rolle, auch wenn Bentele einräumt, dass es fiktionale Elemente durchaus gebe. Sie seien jedoch eher selten zu finden, weil sie sehr schnell zu Unglaubwürdigkeitseffekten und Vertrauensverlusten führen würden (vgl. 1994b: 247). Der fiktionale Charakter scheint sich hier darauf zu beschränken, dass etwas nicht zu überprüfen sei. Durch seine Fixierung auf Realitätsvergleiche interessiert sich das realistische Paradigma fast ausschließlich für - wie auch immer - überprüfbare Beschreibungen, während z. B. Bewertungen, Prognosen oder Gerüchte hier weitgehend ausgeschlossen werden. Damit aber bleiben zentrale Aspekte der PR außen vor.

\section{Die Allmachtsthese im konstruktivistischen Paradigma}

In den Arbeiten, die dem konstruktivistischen Paradigma zugeordnet werden können, nimmt die Frage nach der Wirklichkeit von PR-Konstruktionen die zentrale Stellung ein. Zentraler Bezugspunkt im konstruktivistischen PR-Diskurs sind hier sicherlich die Arbeiten von Merten (u. a. 1992, 2008a, 2008b, 2009, 2010). Daneben gibt es eine Reihe weiterer konstruktivistisch argumentierender bzw. geprägter Ausarbeitungen zur PR 
wie von Jarchow (1992), Kückelhaus (1998), Kocks (2007) oder von Westerbarkey (2003), die zum Teil große Gemeinsamkeiten mit Mertens Arbeiten aufweisen. Wenn im Wesentlichen nur Mertens Perspektive diskutiert wird, ist ein solches Vorgehen auch deshalb eine Verkürzung, weil die konstruktivistisch argumentierende PR-Forschung nur wenige der vielfältigen konstruktivistischen Ansätze berücksichtigt hat.

Was ist im konstruktivistischen Paradigma das zentrale Erfolgskriterium für PR-Beschreibungen? Zentraler Bezugspunkt von Mertens Argumentation ist der "Siegeszug der Fiktion“ (Merten 2008b: 5), der mit der Epoche der Mediengesellschaft begonnen habe. Medien liefern, so Merten (vgl. ebd.: 5; 2008a: 48), keine „reale“ Wirklichkeit mehr, sondern grundsätzlich nurmehr „fiktionale“ Wirklichkeiten: „Die Verhältnisse drehen sich geradezu um: Nicht der ist gut aufgestellt, der wirklich gut aufgestellt ist, sondern der, der in den Medien wirklich gut aufgestellt erscheint: Der Anschein, nicht die realen Fakten erzeugen jetzt die weiteren relevanten Fakten" (ders. 2008b: 5-6). Dieser fiktionalen Strukturen bedient sich PR: „PR ist ein Prozess intentionaler und kontingenter Konstruktion wünschenswerter Wirklichkeiten durch Erzeugung und Befestigung von Images in der Öffentlichkeit“ (ders. 1992: 44; Kückelhaus 1998: 372). Als Ergänzung und Konkretisierung zu früheren Arbeiten von Merten können aktuellere Arbeiten gelesen werden, in denen Merten insbesondere das Wie konkretisiert. Dazu stellt er den Täuschungsbegriff in das Zentrum seiner Überlegungen. „Ist Täuschen notwendig für Public Relations? Und wenn ja, wie weit darf die Täuschung gehen?“ (Merten 2008b: 1). Basis seiner Überlegungen ist ein sehr weiter Täuschungsbegriff. „Da alle Darstellung perspektivisch verfährt und die Perspektive immer nur einen Ausschnitt darstellt, [...] [beginnt Täuschung] bereits mit der Wahl der Perspektive, unter der irgendetwas in der Öffentlichkeit dargestellt wird. Täuschung ist bei aller unabsichtlichen, aber erst recht bei absichtlicher Darstellung also stets dabei, Täuschung ist überall.“ (ebd.: 4) Wenn Täuschung aber nicht einmal an das Kriterium der Intentionalität gebunden wird, wie es bei anderen ebenfalls weiten Definitionen üblich zu sein scheint (vgl. dazu Köhnken 1990: 3), dann ist in der Tat - wie Merten sagt - Täuschung überall. Wenn die Selektivität als Grundannahme sowohl konstruktivistischer als auch systemtheoretischer Überlegungen ja gerade eine spezifische Perspektive impliziert, ist nichts vorstellbar, wo keine Täuschung vorliegt. Welchen erkenntnisbringenden Nutzen aber hat ein solcher voraussetzungsloser Täuschungsbegriff dann noch - außer vielleicht den einer gezielten Provokation?

$P R$-Beschreibungen scheitern - dieser Eindruck drängt sich auf - zumindest in Mertens Sichtweise nahezu gar nicht. Merten kann damit als fast schon euphorischer Vertreter der Allmachtsthese von PR bezeichnet werden. Mal ist PR ein Meta-Kommunikator, der entscheidet, „was, wann, wo, wie und mit welcher gewünschten Wirkung kommuniziert werden soll“" (Merten 1992: 44). Und mal liegt der Vorteil fiktionaler Konstrukte „in ihrer einfachen, schnellen und kostengünstigen Erzeugung und Veränderung“ (ders. 2008b: 6). In seinen Arbeiten scheinen der PR nur selten Grenzen gesetzt zu werden, zumal sich die Mediengesellschaft „tendenziell auf die Nichtnachprüfbarkeit von Behauptungen aller Art eingerichtet“ hat (ders. 1992: 37). Aber wie plausibel ist eine solche Allmachtsthese? Die empirischen Untersuchungen zu den Beziehungen von PR und Journalismus haben gezeigt, dass PR bei seiner Imagekonstruktion durchaus Grenzen gesetzt sind (vgl. Raupp 2008: 204). Und noch grundsätzlicher: Wenn mehrere direkt konkurrierende Organisationen Images kreieren, können nicht alle gleichermaßen erfolgreich sein.

Hinzu kommt bei Merten ein anderer Kritikpunkt, der angesichts der euphorischen Einschätzung von Fiktionen überrascht. So lassen sich an den Stellen, an denen Merten 
Kernbegriffe wie die „reale“ Wirklichkeit definiert (vgl. ders. 1999: 253), Hinweise darauf finden, dass auch in Mertens Verständnis Konstruktionen letztlich doch an einer ontischen Realität überprüft werden können. Damit bestätigt Merten die zentrale Kritik von Mitterer am Konstruktivismus. Dieser unterstellt dem konstruktivistischen - ähnlich wie dem realistischen - Paradigma, dass seine Beschreibungen an der Realität scheitern können. Dies begründet er beim Konstruktivismus mit dem Viabilitätsbegriff. Einerseits werde mit dem Viabilitätsbegriff noch allgemein eine Beziehung des Passens zu bislang konstruierten Wirklichkeiten bezeichnet (vgl. Glasersfeld 1992: 39), andererseits aber ein Scheitern zur Realität beschrieben: „Merkwürdigerweise kann es gerade dann, wenn unsere [...] nichtviablen Konstruktionen misslingen oder scheitern[,] zu einem direkten Kontakt, zu einer Konfrontation mit der ,ontischen Realität' kommen. “ (Mitterer 2001: 122-123) Noch deutlicher wird der zumindest implizite Dualismus mitunter in erkenntnistheoretischen „Grenzfragen“ wie zur Unterscheidung von Fakten und Fiktionen. So versteht Kohring unter einer Fiktion eine subjektiv gebildete Wahrnehmung, für die kein Wahrheits- oder Wirklichkeitsbeweis angestrebt wird und die nicht von jedem genauso wahrzunehmen ist (vgl. Kohring 2002: 91-92). Wie aber sind in Abgrenzung zu einem solchen Verständnis Fakten ohne einen beschreibungsverschiedenen und beobachterunabhängigen Bezug logisch zu bestimmen?

Wie werden im konstruktivistischen Paradigma schließlich Trend-Beobachtungen wie die Fiktionalisierung erklärt? Bei der Analyse von Inszenierungen, der Theatralisierung oder der Fiktionalisierung aus konstruktivistischer Perspektive gerät der Konstruktivismus an eine ganz andere Grenze. Im Konstruktivismus gab es - zumeist implizit - immer schon zwei Konstruktivitäts-Praxen (vgl. Weber 2005: 333). Dies ist erstens die epistemologische Allaussage, dass letztlich alles Konstruktion sei. Zweitens ist es die empirische Trendaussage, dass z. B. Massenmedien immer mehr Wirklichkeit konstruieren. Wenn Trends wie die Fiktionalisierung untersucht werden, verfängt sich eine konstruktivistische Betrachtung schnell in Widersprüchen, weil einerseits immer schon alles Konstruktion war, aber andererseits jetzt noch mehr oder anders konstruiert wird. Bewusste Inszenierungen sind damit zugleich Teil der konstruktivistischen Allund Trendaussage. Der Konstruktivismus befindet sich damit in einem „argumentativen Dilemma“ (Weber 2005: 342).

Damit kann auch das konstruktivistische Paradigma wichtige erkenntnistheoretische Fragestellungen der PR nicht plausibel beantworten. Grundsätzlich bietet eine konstruktivistische gegenüber der realistischen Perspektive aber den zentralen Vorteil, dass der Konstruktionscharakter und damit die spezifische Selektivität von PR herausgestellt wird, mit denen z. B. Unterschiede zu journalistischen Wirklichkeitskonstruktionen herausgearbeitet werden können. Zudem sind hier systemtheoretische Überlegungen und Konzepte wie das der funktionalen Transparenz (vgl. Szyszka 2009: 145-146) anschlussfähig, so dass konstruktivistische Überlegungen sozialtheoretisch erweiterbar sind.

\section{Zwischen All- und Ohnmacht in einer non-dualistischen Perspektive}

Die Diskussion des realistischen und konstruktivistischen Paradigmas hat erstens gezeigt, dass eine erkenntnistheoretische Perspektive problematisch ist, die auf einen Realitätsvergleich nicht verzichtet. Wer kann schon hinter Unternehmensfassaden gucken? Und wer kann abschließend beurteilen, ob die Insider-Berichte eines entlassenen Mitarbeiters „wahrer“ sind als die offiziellen PR-Statements? Begründungsversuche, die auf das „Echte“ und „Wahre“ verweisen, geraten schnell in einen infiniten Regress. Daher 
ist eine erkenntnistheoretische Perspektive vielversprechender, die auf einen Realitätsvergleich verzichtet und strikt im Diesseits verbleibt.

Zweitens ist deutlich geworden, dass dennoch vieles dafür spricht, dass beschriebene Differenzen z. B. zwischen PR-Selbstbeschreibungen und journalistischen Fremdbeschreibungen die zugeschriebene Vertrauenswürdigkeit schwächen können. Offenkundig gibt es Beschreibungen, die tendenziell als vertrauenswürdiger eingeschätzt werden, wenn PR-Selbstbeschreibungen im Widerspruch dazu stehen. Eine erkenntnistheoretische Perspektive, die auf einen Realitätsvergleich verzichtet, muss deutlich machen, wie man zu solchen Vertrauenswürdigkeitszuschreibungen kommt.

Schließlich sind drittens Trend-Beobachtungen wie die Theatralisierung und die Fiktionalisierung - ganz allgemein formuliert - mit der Vermutung verbunden, dass sich die massenmediale Wirklichkeit bzw. deren Produktionsweise verändern. Hier stellt sich die Frage nach alternativen Erklärungsansätzen, die nicht erneut in die erkenntnistheoretische Falle von „wahrer“ und „weniger wahr“ führen.

Im Folgenden sollen die Antworten der non-dualistischen Perspektive auf die oben formulierten Fragen herausgearbeitet werden. Da die non-dualistische Perspektive bislang relativ wenig rezipiert wurde, werden für die Argumentation relevante Grundüberlegungen jeweils kurz erläutert. Dabei ist es das Ziel, die Überlegungen von Mitterer aus seiner sicherlich gewöhnungsbedürftigen Sprache zu „übersetzen“, um die Anschlussfähigkeit an vorhandene Diskurse zu gewährleisten.

\section{Warum bzw. woran scheitern PR-Beschreibungen?}

Es ist oben herausgearbeitet worden, dass im realistischen und selbst im konstruktivistischen Paradigma Thesen an der Realität scheitern. Durch den „Wegfall“ der Realität im Non-Dualismus geht dieser Bezugspunkt verloren. Woran aber können Beschreibungen bzw. Thesen dann noch scheitern? Thesen scheitern an neuen Thesen, die dann wieder als „wahr“ ausgewiesen werden, bis sie an neuen Thesen scheitern (vgl. Weber 2005: 262). Und noch einfacher: „Auffassungen sind wahr, weil und solange wir sie vertreten, und sie sind falsch, weil und solange wir sie nicht vertreten“ (Mitterer 2001: 105). An anderer Stelle arbeitet Mitterer die strikte Verzeitlichung dieser Überlegung noch deutlicher heraus: „Die Unterscheidung zwischen Schein und Sein, zwischen für wahr halten und wahr sein, sind argumentative Unterscheidungen, die das Verhältnis zwischen früheren und gegenwärtigen Auffassungen markieren, zugunsten derer vielleicht die früheren revidiert worden sind“ (Mitterer 2001: 60). Zur „Überprüfung“ von Thesen können (wissenschaftliche) Prüfverfahren entwickelt werden. Basis solcher Prüfverfahren sind eine gemeinsame Ausgangsbasis - mithin jene konsensuellen Beschreibungen, die gegenwärtig nicht Gegenstand der Erörterung sind (vgl. ders. 1992: 83f.). Und im Idealfall ist das Prüfverfahren - also zum Beispiel ein Untersuchungsdesign - ebenfalls selbst konsensuell. Wenn beides gegeben ist, gibt es gute Chancen, dass das Ergebnis auf breiter Basis als „wahr“ ausgezeichnet wird. Wenn Beschreibungen weitgehend konsensuell sind - also nicht mehr oder kaum noch ernsthaft hinterfragt werden -, wird aus ihnen so etwas wie eine neutralistische Ausgangsbasis bzw. ein Basiskonsens für weitere Beschreibungen (ebd.: 71-75). Dies kann auch als Wabrheit bezeichnet werden. Dazu zählen Auffassungen, „die wir vertreten müssen, um in unserer Gesellschaft überleben zu können“ (ders. 2001: 106).

Hier zeigt sich die Kontingenz bzw. Willkür von Beschreibungen. Wenn die ontische Realität als Vergleichsmaßstab ausfällt, folgt daraus die Frage, wie erklärt werden kann, warum Beschreibungen scheitern bzw. die einen Beschreibungen anderen Beschreibungen vorgezogen werden. Dies und die Konsequenzen dieser allgemeinen Überlegungen 
für PR-Beschreibungen werden im Kontext der Erfolgskriterien für PR-Beschreibungen herausgearbeitet.

\section{Was sind die Erfolgskriterien für PR-Beschreibungen?}

Was entscheidet nun in einem erkenntnistheoretischen Gedankengebäude ohne Realitätsvergleich darüber, ob Beschreibungen so far zur (positiven) Grundlage für Beschreibungen from now on werden? Und: Wie ist die empirisch zu beobachtende Evidenz zu erklären, dass z. B. journalistische Beschreibungen in der Regel länger vertreten werden als PR-Beschreibungen? Hierzu trägt insbesondere der Selbstdarstellungscharakter von PR-Beschreibungen bei, die wie jede Selbstbeschreibung in der Regel als weniger glaubwürdig und vertrauenswürdig eingeschätzt werden als Fremdbeschreibungen wie journalistische Medienberichte. Auf welcher Grundlage aber entscheidet man über Vertrauenswürdigkeitszuschreibungen - wenn nicht durch einen Realitätsvergleich? Im Folgenden soll die These ausgeführt werden, dass hierzu - ähnlich wie bei Bentele - ein Vergleich z. B. von PR-Beschreibungen und Medienbeschreibungen durchgeführt wird, der - im Gegensatz zu Bentele - strikt im Diesseits verbleibt und damit auf jeglichen Realitätsvergleich verzichtet. Diese als Vergleichsmaßstab dienenden Beschreibungen, die allesamt tendenziell als vertrauenswürdiger als PR-Beschreibungen bewertet werden, können (a) Beschreibungen im Sinne eigener Erfahrungen bzw. Beobachtungen, (b) Beschreibungen von persönlich bekannten Personen oder aber (c) andere Medienbeschreibungen sein (vgl. Merten 1999: 249).

Beschreibungen im Sinne eigener Erfahrungen (a) gelten als besonders relevant für Fragen der Vertrauenswürdigkeitseinschätzung oder von Imageveränderungen (vgl. ebd.: 249). Während im realistischen und konstruktivistischen Paradigma zwischen dem Objekt und der Beschreibung unterschieden wird, interessiert sich der Non-Dualismus für jenseitige Objekte nicht: Das Objekt der Beschreibung ist nicht beschreibungs- oder „sprachverschieden“, sondern ist jener Teil der Beschreibung, der bereits ausgeführt worden ist (vgl. Mitterer 1992: 56). Mit anderen Worten: „Einen Apfel beschreiben, heißt die (bereits ausgeführte) Beschreibung /ein Apfel/ fortsetzen“ (ebd.). Daher sind auch jene Beschreibungen im Sinne eigener Erfahrungen nichts anderes als die Fortführung bereits vorliegender Beschreibungen. Mithin werden z. B. PR-Beschreibungen nicht mit einer direkt zugänglichen beschreibungsverschiedenen Welt verglichen, wie es Benteles Formulierung einer Isomorphiebeziehung von Medienbeschreibungen und Ereignissen nahelegt (vgl. Bentele 1992a: 164). Vielmehr werden hier PR-Beschreibungen mit „eigenen" Beschreibungen verglichen. Auch wenn einiges dafür spricht, dass in vielen Fällen „eigene“ Beschreibungen als vertrauenswürdiger und damit als Basis-Konsens ausgewiesen werden, so sind letztlich beide Beschreibungen kontingent. Die non-dualistische Perspektive ermöglicht damit auch einen neuen Blick auf die in der PR so oft genannte normative Anforderung der Übereinstimmung von Wort und Tat (vgl. z. B. Hundhausen 1951: 160-161). In dem hier vorgestellten Gedankengebäude sind das „Wort“ z. B. PRBeschreibungen und die „Tat“ eigene Beschreibungen im Sinne eigener Erfahrungen oder Beschreibungen von „Betroffenen“, also z. B. entlassenen Mitarbeitern. Letztlich werden auch hier Beschreibungen miteinander verglichen.

Ein solcher Vergleich zwischen mehreren Beschreibungen liegt letztlich auch in den anderen beiden Fällen vor - sowohl bei Beschreibungen von persönlich bekannten Personen (b) als auch im Falle von Medienbeschreibungen (c). So könnte man im letzteren Fall eine PR-Beschreibung überprüfen, indem man zum selben Thema die Berichterstattung einer Zeitung liest. Wenn die PR-Beschreibung bestätigt wird, könnte dies dazu führen, dass sie als neuer Basiskonsens ausgewiesen wird (vgl. Weber 2005: 318). Insbe- 
sondere journalistische Berichte haben im Gegensatz zu PR-Beschreibungen den enormen Vorteil, dass sie eben Fremd- und keine Selbstbeschreibungen sind und mithin tendenziell als vertrauenswürdiger beschrieben werden. PR dürfte sich daher in der Mehrzahl der Fälle insbesondere an der journalistischen Berichterstattung orientieren - weil ein Abweichen davon als vertrauensunwürdig und damit höchst riskant erscheinen kann. Hier wird deutlich, dass PR sich mit ihren PR-Beschreibungen tendenziell umso mehr von dem entfernen kann, was sie selbst als wahr bezeichnen würde, je weniger die beschriebenen Themen für externe Bezugsgruppen erfahrbar sind und je mehr das konkrete Unternehmen in der Vergangenheit in hohem Maße als vertrauenswürdig bezeichnet wurde.

Damit ist deutlich geworden, dass Vertrauenswürdigkeit das zentrale Erfolgskriterium für PR-Beschreibungen ist. PR-Beschreibungen scheitern vor allem an fehlender Vertrauenswürdigkeit - oder genauer: weil sie seltener als vertrauenswürdig bewertet werden als z. B. journalistische Beschreibungen. Das Problem der Vertrauenswürdigkeit ist hier auf verschiedenen Ebenen relevant: Zunächst geht es grundsätzlich um einen Vertrauenswürdigkeitswettbewerb zwischen den PR-Selbstbildern und journalistischen Fremdbildern, hinzu kommt eine Wettbewerbssituation zwischen PR-Beschreibungen unterschiedlicher Organisationen. Vertrauenswürdigkeit ist damit in einer non-dualistischen Perspektive der zentrale erkenntnistheoretische Bezugspunkt von PR. In einer non-dualistischen Perspektive problematisiert PR statt Wabrbeit nur die - vermutete bzw. unterstellte - Vertrauenswürdigkeit von Beschreibungen. PR interessiert sich nur dafür, welche Chancen eine Beschreibung ibrer Einschätzung nach hat, von den relevanten Bezugsgruppen als vertrauenswürdig bezeichnet zu werden. Mit Huck kann PR damit als organisationaler „Glaubwürdigkeits-Gatekeeper“ (Huck 2006: 50) bzw. etwas weiter als Vertranenswürdigkeits-Gatekeeper ${ }^{1}$ verstanden werden, allerdings ohne jegliche Prüfung eines ontischen Wahrheitsgehaltes. PR wird dazu neben den oben skizzierten „konkurrierenden“ Beschreibungen auch die Aspekte berücksichtigen, die in der Glaub- bzw. Vertrauenswürdigkeitsforschung verschiedentlich skizziert wurden (vgl. Hovland et al. 1953, Köhnken 1990), zu denen aber sicherlich weiterer Forschungsbedarf besteht. Dazu zählen z. B. neben der Konsistenz von Beschreibungen die Berücksichtigung von Gegenargumenten oder Fragen des Auftritts.

Die Vertrauenswürdigkeit wäre aus einer systemtheoretischen Perspektive nicht das primäre Selektionskriterium, sondern nach der Leit-Unterscheidung wie z. B. der Legitimation auf der Programm-Ebene zu verorten. Die Einschätzung zur Vertrauenswürdigkeit ist aus einer erkenntnistheoretischen Perspektive die zentrale Leistung von PR. PR prüft dazu vorliegende Beschreibungen daraufhin, ob sie von Bezugsgruppen als vertrauenswürdig eingeschätzt werden könnten. Damit versucht PR, Vertrauenswürdigkeitszuschreibungen der Organisation zu sichern. Noch einmal: Wenn man PR als organisationalen Vertrauenswürdigkeits-Gatekeeper konzipiert, ändert dies weder etwas daran, dass der Journalismus PR-Beschreibungen seinerseits prüft und vielfach als vertrauensunwürdig einschätzt (vgl. Weischenberg/Malik/Scholl 2006: 127), noch dass journalistische Beschreibungen in der Regel als vertrauenswürdiger eingeschätzt werden. PR versucht mit dem Vertrauenswürdigkeits-Gatekeeping, die Wahrscheinlichkeit von Vertrauenswürdigkeitszuschreibungen zu erhöhen - nicht mehr und nicht weniger.

Das einleitende Beispiel zur Versenkung der Brent Spar zeigt noch einmal, warum die vermuteten Chancen bei Vertrauenswürdigkeitszuschreibungen durch Bezugsgrup-

1 Während sich Glaubwürdigkeitszuschreibungen auf die Angemessenheit von Fakten beschränken, beziehen sich Vertrauenswürdigkeitszuschreibungen auf eine Beschreibung allgemein (vgl. Kohring 2004). 
pen für PR entscheidend sind. Damals ist lange Zeit eine Beschreibung als unglaubwürdig bezeichnet worden, die heute den Status der "Wahrheit“ genießt. PR-Beschreibungen scheitern mithin nicht, weil der Pressesprecher nicht genau genug hingeschaut hätte oder weil eine PR-Konstruktion wegen fehlender Viabilität „an die Wand“ gestoßen wäre, sondern schlicht und ergreifend: weil eine PR-Beschreibung als nicht vertrauenswürdig und damit konsensuell eingeschätzt wurde und deshalb keine positive Grundlage für künftige Beschreibungen war. PR kann nur prüfen und entsprechend auswählen, was von den Rezipienten als vertrauenswürdig eingeschätzt werden könnte.

Es ist gezeigt worden, dass in einer non-dualistischen Perspektive Vertrauenswürdigkeit das zentrale Erfolgskriterium ist. Zwar wird auch im konstruktivistischen Paradigma und insbesondere in realistischen Arbeiten von Bentele die Relevanz von Vertrauen bzw. Vertrauenswürdigkeit für PR betont. Zugleich aber wird in einer realistischen Perspektive die Relevanz von Vertrauen durch die Möglichkeit von Realitätsvergleichen geschwächt: Wie wichtig ist noch Vertrauen, wenn das Erkennen von Realität Gewissheit verschafft? Zudem gehen die meisten Arbeiten, die die Relevanz von Vertrauen betonen, von einem anderen Beziehungsverhältnis zwischen PR und Vertrauen aus. Während hier Vertrauen in PR bzw. vertrauenswürdige PR thematisiert werden, interessiert sich z. B. Bentele vor allem für Vertrauen durch PR in Organisationen (vgl. Hoffjann 2011).

\section{Wie werden im Non-Dualismus Trend-Beobachtungen wie die Fiktionalisierung erklärt?}

Man könnte vermuten, dass klassische Dualitätspaare wie Sein und Schein, Realität und Irrealität, Fakten und Fiktionen in einer non-dualistischen Perspektive keinen Platz hätten. Das hätte zur Folge, dass auch Trend-Beobachtungen wie die Fiktionalisierung oder die Zunahme von Inszenierungen nicht zu erklären wären. In einer non-dualistischen Perspektive kann dieses Problem so gelöst werden, indem sie uminterpretiert werden: Sein, Realität und Fakten beziehen sich nicht auf beschreibungsverschiedene Objekte, sondern werden als Basiskonsens verstanden. Beide Seiten der Unterscheidungen bleiben immer im Diskursdiesseits (vgl. Weber 2005: 340-341). Folglich sind es nur differente Modi von Beschreibungen: Während mit real und faktisch konsensuelle - also im oben erläuterten Verständnis „wahre“ - Beschreibungen bezeichnet werden, sind fiktionale Beschreibungen noch nicht konsensuell. Alle Beschreibungen schließen jedoch immer an alte Beschreibungen an, und diese neuen Beschreibungen können dann mit Attributen wie fiktional etc. ausgestattet werden (vgl. ebd.: 341).

Wie können nun Trend-Beobachtungen wie die Fiktionalisierung bzw. die zunehmenden Inszenierungen erklärt werden? Im Gegensatz zum Konstruktivismus, der zwischen der konstruktivistischen All- und Trendaussage unterscheidet, interessiert sich der Non-Dualismus nur für die Trendaussage (vgl. ebd.: 337-350). Fiktionalisierung wäre dann nichts anderes als eine Zunahme von noch nicht konsensuellen Beschreibungen. Und die damit in Verbindung gebrachte Klage einer Entfernung von der Wirklichkeit wäre in einer non-dualistischen Rekonstruktion nichts anderes als eine Reaktion auf Kontingenzerfahrungen, die durch eine Vielzahl von neuen Beschreibungen gemacht werden, die immer schneller aufgestellt werden (vgl. ebd.: 340). Mit jeder neuen Medienbeschreibung entfernt man sich von der Wirklichkeit - also der ursprünglichen Beschreibung so far (vgl. ebd.: 318).

Zudem können empirische Inszenierungs- und Konstruktivitätsgrade widerspruchsfreier beschrieben werden. Dazu ein konkretes Beispiel: Man könnte Konstruktivitätsgrade von Veranstaltungen empirisch untersuchen, indem man eine Befragung von Veranstaltungsmachern mit einer Beobachtung der Veranstaltung kombiniert. Dabei könn- 
ten bewusste Konstruktivitätsgrade bzw. Inszenierungsgrade für PR-Inszenierungen untersucht werden, ähnlich wie Weber (1999) dies für den Journalismus getan hat. Als Indikatoren könnten gelten: die inhaltliche Vorbereitung der Statements, die Festlegung des Ablaufs, des Medienbildes und der Perspektive der Foto- und TV-Kameras etc. Bei einem Vergleich von zwei Veranstaltungen könnte man dann zu dem Ergebnis kommen, dass Veranstaltung A in höherem Maße inszeniert wurde als Veranstaltung B. Ein solches Ergebnis ist keine erneute Dualisierung von jenseitiger Realität vs. diesseitiger Inszenierung. Das heißt folglich auch, dass es keine Annäherung an die „wahre“Veranstaltung gibt. Wenn man in einem zweiten Schritt zwischen der Produktions- und Rezeptionsseite unterscheidet, können die jeweiligen Beschreibungen miteinander verglichen werden: Auf der Produktionsseite ist es die (Nicht-)Inszenierung eines Ereignisses, auf der Rezipientenseite ist es die (Nicht-)Zuschreibung von Authentizität durch Beobachter. Um dies zu untersuchen, könnte man die oben skizzierte Untersuchungsanlage um Befragungen von Zuschauern $\mathrm{zu}$ ihrer Einschätzung nach der Zuschreibung von (In-)Authentizität befragen. Entsprechend kann der Zusammenhang zwischen Inszenierungsgraden und der Beschreibung der Rezipienten untersucht werden. Mögliche Ergebnisse einer solchen Untersuchung könnten sein: PR-Beschreibungen mit einem hohem Inszenierungsgrad werden von Rezipienten als inszeniert beschrieben. Oder aber: PR-Beschreibungen mit einem hohem Inszenierungsgrad werden von Rezipienten als nicht-inszeniert/authentisch beschrieben. Das zweite Ergebnis würde dann die in den vergangenen Jahren vielfach geäußerte These von der Authentizität durch Inszenierung stärken (vgl. Fischer-Lichte et al. 2007).

Wie verhält sich ein solches non-dualistisches Verständnis von PR zur realistischen Ohnmachtsthese und zur konstruktivistischen Allmachtsthese? In einer non-dualistischen Perspektive geraten Chancen und Risiken gleichermaßen in den Blick (vgl. Tab.). Chancen ergeben sich, wenn PR erfolgreich Beschreibungen etabliert, die sie selbst als „fiktiv“ bezeichnen würde. Risiken zeigen sich, weil PR beispielsweise in Krisenzeiten nur geringe Chancen haben dürfte, eine aus ihrer Perspektive falsche Beschreibung zu korrigieren. So ist es in diesem Verständnis nicht überraschend, dass ein Pressesprecher auch mit einer PR-Beschreibung dem Lügen-Vorwurf ausgesetzt werden kann, wenn er sie nach bestem Wissen und Gewissen angefertigt hat. Genau deshalb werden hier nicht das „beste Wissen und Gewissen“ als Selektionskriterium, sondern die bei Rezipienten vermuteten Vertrauenswürdigkeitschancen favorisiert. Im realistischen Verständnis wäre dies kaum vorstellbar.

Daher ist auch eine weitere zentrale Annahme des Non-Dualismus höchst ambivalent für die PR. Mitterer setzt dem Streben nach Wahrheit von Konstruktivismus und Realismus ein Streben nach Wechsel entgegen (vgl. Mitterer 1992: 110). Die Plausibilität dieses Gedankens belegen virale Spots im Web 2.0: Lange wurden Spots mit verwackelten und unscharfen Bildern als authentisch beschrieben. Nachdem in Medienberichten mehrfach über die (kommerziellen) Hintergründe solcher Spots berichtet wurde, dürfte sich hier die Authentizitätszuschreibung verändern. Ein zweites Beispiel sind die wechselnden journalistischen Berichterstattungen zu politischen Themen oder zur Einschätzung von Personen über einen längeren Zeitraum hinweg. Hier scheint ein journalistisches Streben nach Wechsel die Kommentierungszyklen ebenfalls deutlich plausibler zu 
Tabelle: Annabmen verschiedener erkenntnistheoretischer Perspektiven zur PR

\begin{tabular}{|l|l|l|l|}
\hline & $\begin{array}{l}\text { Realistische } \\
\text { Perspektive }\end{array}$ & $\begin{array}{l}\text { Konstruktivistische } \\
\text { Perspektive }\end{array}$ & $\begin{array}{l}\text { Non-dualistische } \\
\text { Perspektive }\end{array}$ \\
\hline $\begin{array}{l}\text { Zentrales Erfolgs- } \\
\text { kriterium für PR- } \\
\text { Beschreibungen }\end{array}$ & Wahrheit & Fiktionalität & Vertrauenswürdigkeit \\
\hline $\begin{array}{l}\text { Woran scheitern } \\
\text { PR-Beschreibun- } \\
\text { gen? }\end{array}$ & An der Realität & An fehlender Viabilität & $\begin{array}{l}\text { An anderen als vertrau- } \\
\text { enswürdiger bezeichne- } \\
\text { ten Beschreibungen }\end{array}$ \\
\hline $\begin{array}{l}\text { Wie wird der Ein- } \\
\text { fluss von PR be- } \\
\text { wertet? }\end{array}$ & $\begin{array}{l}\text { Obnmachtsthese } \\
\text { Geringer Einfluss, da } \\
\text { PR-Beschreibungen an } \\
\text { der Realität überprüft } \\
\text { werden können (vgl. } \\
\text { Bentele 1994b: 254) }\end{array}$ & $\begin{array}{l}\text { Allmachtsthese } \\
\text { Großer Einfluss, da sich } \\
\text { die Mediengesellschaft } \\
\text { auf die Nichtnachprüf- } \\
\text { barkeit von Beschreibun- } \\
\text { gen eingestellt hat (vgl. } \\
\text { Merten 1992: 37) }\end{array}$ & $\begin{array}{l}\text { Zwischen Ohnmachts- } \\
\text { und Allmachtsthese } \\
\text { Geringer und großer Ein- } \\
\text { fluss gleichermaßen, da } \\
\text { von fiktionalen Beschrei- } \\
\text { bungen sein kann }\end{array}$ \\
\hline
\end{tabular}

erklären als ein Streben nach Wahrheit. ${ }^{2}$ Ambivalent ist dieses Streben nach Wechsel, weil PR einerseits mit neuen überraschenden Beschreibungen erfolgreich sein kann, andererseits aber auch „Opfer“ journalistischer Herdentriebe (vgl. Ruß-Mohl 2009) werden kann.

\section{Fazit}

In dem Beitrag sind die Antworten der drei erkenntnistheoretischen Kandidaten auf die Fragen zur Wirklichkeit der PR herausgearbeitet worden. Im realistischen Paradigma erscheint die Möglichkeit von Realitätsvergleichen wenig plausibel. Daraus folgt eine weitgehend ohnmächtige PR. Überspitzt könnte man fragen, ob es überhaupt noch der PR bedarf, wenn wir alle die Wahrheit erkennen könnten. Hinzu kommt im realistischen Paradigma, dass sie sich für relevante Trend-Beobachtungen wie Fiktionalisierung und Theatralisierung kaum interessiert. Diese nehmen im konstruktivistischen Paradigma zwar eine zentrale Stellung ein, allerdings befindet sich der Konstruktivismus zwischen seiner epistemologischen Allaussage, dass letztlich alles Konstruktion sei, und der empirischen Trendaussage, dass z. B. Massenmedien immer mehr konstruieren, in einem „argumentativen Dilemma“ (Weber 2005: 342). Zudem schließt das konstruktivistische Viabilitäts-Konzept Realitätsvergleiche in letzter Konsequenz nicht aus. Insbesondere diese Kritik muss bei den Arbeiten Mertens überraschen, da er ansonsten kaum Grenzen für die PR-Wirklichkeitskonstruktionen sieht.

Solche Probleme vermeidet die non-dualistische Perspektive, indem sie sich für eine ontische Realität nicht interessiert, sondern nur auf diesseitige Beschreibungen fokussiert. Daraus folgt die völlige Kontingenz jeglicher Beschreibungen. Es ist ausgeführt worden, dass in dieser Situation die Vertrauenswürdigkeit eine zentrale Bedeutung gewinnt. Da journalistische Beschreibungen in der Regel als deutlich vertrauenswürdiger

2 In systemtheoretischen Beschreibungen des Journalismus ist dieses Streben nach Wechsel statt nach Wahrheit zumindest implizit schon lange erkannt worden. Journalismus berichtet z. B. eben nicht „objektiv“ über technische Risiken, sondern allein nach journalistischen Kriterien (z. B. Görke 1999). 
bewertet werden als PR-Beschreibungen, orientiert sich PR in hohem Maße an ihnen. PR interessiert sich nur dafür, welche Chancen eine Beschreibung ihrer Einschätzung nach hat, von den relevanten Bezugsgruppen als vertrauenswürdig bezeichnet zu werden. Mit dem Bezugspunkt Vertrauenswürdigkeit kann zudem erklärt werden, warum sich PR mit ihren Beschreibungen mitunter allmächtig und mitunter ohnmächtig fühlt. Abschließend ist herausgearbeitet worden, wie Trend-Beobachtungen wie die Fiktionalisierung oder Theatralisierung in einer non-dualistischen Perspektive erklärt und empirisch untersucht werden können. Durch den Vergleich von Beschreibungen und den Verzicht auf die epistemologische Allaussage des Konstruktivismus, dass letztlich alles Konstruktion sei, können diese Phänomene deutlich widerspruchsfreier erklärt werden.

Diesen Vorteilen einer non-dualistischen Perspektive stehen ohne Zweifel Nachteile gegenüber. Da die Zahl non-dualistischer Beiträge noch überschaubar ist, ist eine Vielzahl an Fragen ungeklärt. Schmidt mahnt die Definition zentraler Begriffe wie Sprache, Beschreibung und Diskurs ebenso an wie die Berücksichtigung nichtsprachlicher Handlungen (vgl. Schmidt 2011: 436-437). Zudem hat die Anwendung des Non-Dualismus auf die PR die Relevanz sozialtheoretischer Anknüpfungen gezeigt. Hier scheint der Non-Dualismus ähnlich wie der Konstruktivismus z. B. direkt an systemtheoretische Überlegungen anknüpfen zu können. Dies zeigt, dass ein non-dualistisches Projekt anschlussfähig ist und mithin auf zahlreiche Erkenntnisse der PR-Forschung zurückgreifen könnte, auch wenn in Teilen sicherlich leichte Uminterpretationen notwendig wären.

\section{Literatur}

Baum, Achim / Schmidt, Siegfried J. (2002) (Hrsg.): Fakten und Fiktionen. Über den Umgang mit Medienwirklichkeiten. Konstanz.

Bentele, Günter (1992a): Ethik der Public Relations als wissenschaftliche Herausforderung. In: Avenarius, Horst / Armbrecht, Wolfgang (Hrsg.): Ist Public Relations eine Wissenschaft. Eine Einführung. Opladen: 151-170.

Bentele, Günter (1992b): Ethik der Public Relations als wissenschaftliche Herausforderung. In: PRMagazin, 5: 37-44.

Bentele, Günter (1994a): Öffentliches Vertrauen - normative und soziale Grundlage für Public Relations. In: Armbrecht, Wolfgang / Zabel, Ulf (Hrsg.): Normative Aspekte der Public Relations. Grundlegende Fragen und Perspektiven. Eine Einführung. Opladen: 131-158.

Bentele, Günter (1994b): Public Relations und Wirklichkeit. Beitrag zu einer Theorie der Öffentlichkeitsarbeit. In: Ders. / Hesse, Kurt (Hrsg.): Publizistik in der Gesellschaft. Festschrift für Manfred Rühl. Konstanz: 237-267.

Bentele, Günter (2005): Rekonstruktiver Ansatz der Public Relations. In: Ders. / Fröhlich, Romy / Szyszka, Peter (Hrsg.): Handbuch der Public Relations. Wissenschaftliche Grundlagen und berufliches Handeln. Mit Lexikon. Wiesbaden: 147-160.

Bentele, Günter (2008): Objektivität und Glaubwürdigkeit: Medienrealität rekonstruiert. Herausgegeben von Stefan Wehmeier, Howard Nothhaft und René Seidenglanz. Wiesbaden.

Bentele, Günter (2009): Ethik der Public Relations. Grundlagen, Probleme und Herausforderungen. In: Avenarius, Horst / Bentele, Günter (Hrsg.): Selbstkontrolle im Berufsfeld Public Relations. Reflexionen und Dokumentation. Wiesbaden: 18-47.

Bentele, Günter (2010): Verständigungsorientierte Öffentlichkeitsarbeit. Herausforderungen der PR-Ethik. In: Hömberg, Walter / Hahn, Daniela / Schaffer, Timon B. (2010): Kommunikation und Verständigung. Theorie, Empirie, Praxis. Wiesbaden: 75-94.

Bentele, Günter / Seidenglanz, René (2004): Das Image der Image-Macher. Eine repräsentative Studie zum Image der PR-Branche in der Bevölkerung und eine Journalistenumfrage. Leipzig.

Fischer-Lichte, Erika / Horn, Christian / Pflug, Isabel / Warstat, Matthias (2007) (Hrsg.): Inszenierung von Authentizität (2. Aufl.). Tübingen, Basel.

Glasersfeld, Ernst von (1992): Konstruktion der Wirklichkeit und des Begriffs der Objektivität. In: Gumin, Heinz / Mohler, Armin (Hrsg.): Einführung in den Konstruktivismus. München: 9-39. 
Görke, Alexander (1999): Risikojournalismus und Risikogesellschaft. Sondierung und Theorieentwurf. Opladen, Wiesbaden.

Hoffjann, Olaf (2011): Vertrauen in Public Relations. In: Publizistik, 55, 1: 65-84.

Hovland, Carl I. / Janis, Irving L. / Kelley, Harold H. (1953): Communication and Persuasion. Psychological Studies of Opinion Change. New Haven.

Huck, Simone (2006): Glaubwürdigkeit: Erfolgsfaktor für die Unternehmenskommunikation. Ergebnisse einer qualitativen Befragung von Kommunikationsverantwortlichen. Stuttgart.

Hundhausen, Carl (1951): Werbung um öffentliches Vertrauen. Public Relations. Essen.

Jarchow, Klaus (1992): Wirklichkeiten, Wahrheiten, Wahrnehmungen: Systemtheoretische Voraussetzungen der Public Relations. Bremen.

Kocks, Klaus (2007): Authentische PR als Paradoxon. Essay zur Kunst der Fälschung. Bristol.

Köhnken, Günter (1990): Glaubwürdigkeit. Untersuchungen zu einem psychologischen Konstrukt. München.

Kohring, Matthias (2002): Fakten ins Töpfchen, Fiktionen ins Kröpfchen? Warum Vertrauen in Journalismus mehr ist als Glaubwürdigkeit. In: Baum, Achim / Schmidt, Siegfried J. (Hrsg.): Fakten und Fiktionen. Über den Umgang mit Medienwirklichkeiten. Konstanz: 90-100.

Kohring, Matthias (2004): Vertrauen in Journalismus. Theorie und Empirie. Konstanz.

Kückelhaus, Andrea (1998): Public Relations. Die Konstruktion von Wirklichkeit. Kommunikationstheoretische Annäherungen an ein neuzeitliches Phänomen. Opladen, Wiesbaden.

Kunczik, Michael (2002): Public Relations. Konzepte und Theorien (4. Aufl.). Köln.

Merten, Klaus (1992): Begriff und Funktion von Public Relations. In: PR-Magazin, 23, 11: 35-46.

Merten, Klaus (1999): Einführung in die Kommunikationswissenschaft. Band 1: Grundlagen der Kommunikationswissenschaft. Münster.

Merten, Klaus (2006): Nur wer lügen darf, kann kommunizieren. In: Pressesprecher, 1: 22-25.

Merten, Klaus (2008a): Zur Definition von Public Relations. In: Medien \& Kommunikationswissenschaft, 56,1: 42-59.

Merten, Klaus (2008b): Public Relations - die Lizenz zu Täuschen? In: PR-Journal, http://www.prjournal.de/images/stories/downloads/merten-vortrag\%20muenster\%2019.6..pdf [4.11.2012].

Merten, Klaus (2009): Zur Theorie der PR-Theorien. Oder: Kann man PR-Theorien anders als systemisch modellieren? In: Röttger, Ulrike (Hrsg.): Theorien der Public Relations. Grundlagen und Perspektiven der PR-Forschung (2. Aufl.). Wiesbaden: 51-70.

Merten, Klaus (2010): Ethik der PR: Ethik oder PR für PR. In: Hömberg, Walter / Hahn, Daniela / Schaffer, Timon B. (2010): Kommunikation und Verständigung. Theorie, Empirie, Praxis. Wiesbaden: 95-118.

Merten, Klaus (2011): Scharfe Rügen für den Deutschen PR-Rat. Sehr offener Brief an die PRMänner Avenarius und Gaul. In: http://www.pr-journal.de/images/stories/download2/merten \%20ethik\%20an\%20pr-rat\%2007.01.2011.pdf [4.11.2012].

Mitterer, Josef (1992): Das Jenseits der Philosophie. Wider das dualistische Erkenntnisprinzip. Wien.

Mitterer, Josef (2001): Die Flucht aus der Beliebigkeit. Frankfurt am Main.

Raupp, Juliana (2008): Determinationsthese. In: Bentele, Günter / Fröhlich, Romy / Szyszka, Peter (Hrsg.): Handbuch der Public Relations. Wissenschaftliche Grundlagen und berufliches Handeln. Mit Lexikon (2. Aufl.). Wiesbaden: 192-208.

Riegler, Alexander / Weber, Stefan (2008) (Hrsg.): The Non-Dualizing Philosophy of Josef Mitterer. Constructivist Foundations (Special Issue), $3,3$.

Riegler, Alexander / Weber, Stefan (2010) (Hrsg.): Die Dritte Philosophie. Kritische Beiträge zu Josef Mitterers Non-Dualismus. Weilerswist.

Rosengren, Karl Erik (1979): Bias in the News: Methods and Concepts. In: Studies of Broadcasting, 15: 31-45.

Ruß-Mohl, Stephan (2009): Kreative Zerstörung. Niedergang und Neuerfindung des Zeitungsjournalismus in den USA. Konstanz.

Schmidt, Siegfried J. (2003): Geschichten \& Diskurse. Abschied vom Konstruktivismus. Hamburg.

Schmidt, Siegfried J. (2010): Die Endgültigkeit der Vorläufigkeit. Prozessualität als Argumentationsstrategie. Weilerswist. 
Schmidt, Siegfried (2011): Der Abschied von der Wahrheit. In: Pörksen, Bernhard (Hrsg.): Schlüsselwerke des Konstruktivismus. Wiesbaden: 425-439.

Scholl, Armin (2002): Einleitung. In: Ders. (Hrsg.): Systemtheorie und Konstruktivismus in der Kommunikationswissenschaft. Konstanz: 7-18.

Scholl, Armin (2011). Konstruktivismus und Methoden in der empirischen Sozialforschung. In: Medien \& Kommunikationswissenschaft, 59, 2: 161-179.

Schulz, Winfried (1990): Die Konstruktion von Realität in den Nachrichtenmedien: Analyse der aktuellen Berichterstattung (2. Aufl.). Freiburg, München.

Shell (1995): Die Ereignisse um Brent Spar in Deutschland. Darstellung und Dokumentation mit Daten und Fakten. Die Hintergründe und Einflussfaktoren. Kommentare und Medienresonanzen. Hamburg.

Szyszka, Peter (2009): Organisation und Kommunikation: Integrativer Ansatz einer Theorie zu Public Relations und Public Relations-Management. In: Röttger, Ulrike (Hrsg.): Theorien der Public Relations. Grundlagen und Perspektiven der PR-Forschung (2. Aufl.). Wiesbaden: 135-150.

Vorfelder, Jochen (1995): Brent Spar oder die Zukunft der Meere. Ein Greenpeace-Report. München.

Weber, Stefan (1999): Wie journalistische Wirklichkeiten entstehen. Salzburg.

Weber, Stefan (2005): Non-dualistische Medientheorie. Eine philosophische Grundlegung. Konstanz.

Weischenberg, Siegfried / Malik, Maja / Scholl, Armin (2006): Die Souffleure der Mediengesellschaft. Report über die Journalisten in Deutschland. Konstanz.

Westerbarkey, Joachim (2003): Maskierung und Beeinflussung. Die gesellschaftlichen Eliten und die Verschleierung der Macht. In: Hettlage, Robert (Hrsg.): Verleugnen, Vertuschen, Verdrehen. Leben in der Lügengesellschaft. Konstanz: 199-214.

Westerbarkey, Joachim (2004): Illusionsexperten. Die gesellschaftlichen Eliten und die Verschleierung der Macht. In: Raupp, Juliana / Klewes, Joachim (Hrsg.): Quo vadis Public Relations? Auf dem Weg zum Kommunikationsmanagement. Bestandsaufnahmen und Entwicklungen. Wiesbaden: $30-41$. 